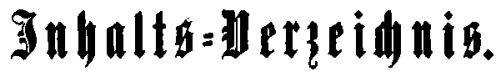

\section{(Erfter đeil.}

Einleitung.

A. Iraftloserthärung abhanben getommener oder bernichteter $\mathfrak{u}$, hunden im Aufgebotaberfahren

B. Bahlungsiperre

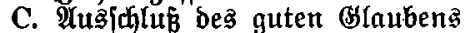

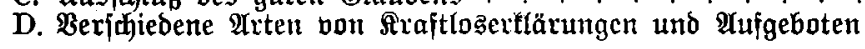

\section{Imeiter Oril.}

Die 8uläfigleit ber firaftozerfärang bai bca ber. idiebenen $\mathfrak{a}$ ten bon $\mathfrak{u}_{\mathfrak{t}}$ unden.

1. $\mathfrak{A}$ b ॠ

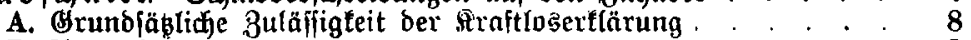

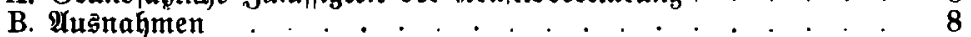

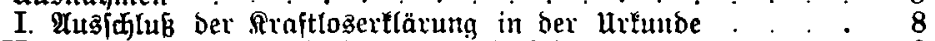

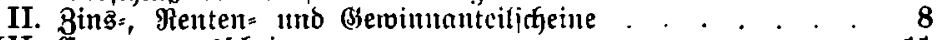

III. Exneuerungsjheine . . . . . . . . . . . . . . . 11

IV. Bantnoten . . . . . . . . . . . . . . . . . 12

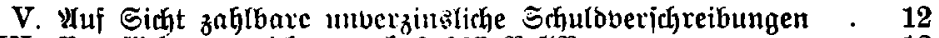

VI. Berpflicfitungszeidien nadj $\$ 807 \mathfrak{B}$ (SB. . . . . . . 13

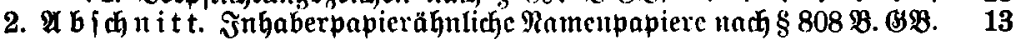

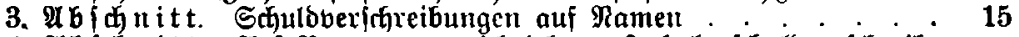

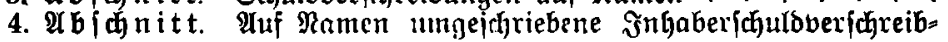
ungen . . . . . . . . . 16

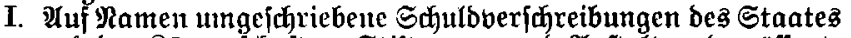

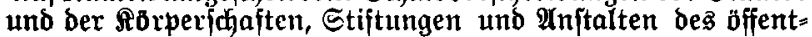
lichen Redhtes . . . . . . . . . . . 16

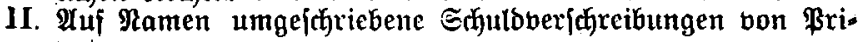
batent

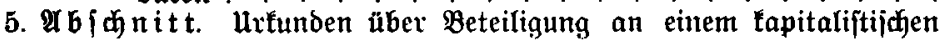
Unternebmen . . . . . . . . . 18

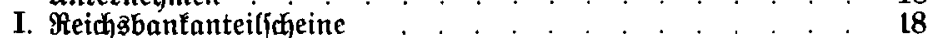

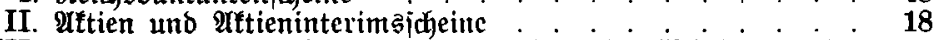

III. Anteilfujeinc über Beteiligung an einer Bejedichaft mit be=

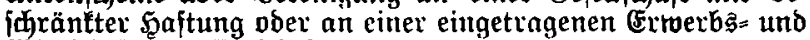

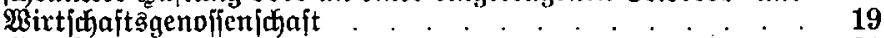

IV. Senufifheine . . . . . . . . . . . . . . . . . 21

V. Tuxifheine

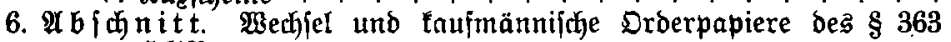
E(SOB. . . . . . . . . . . 22

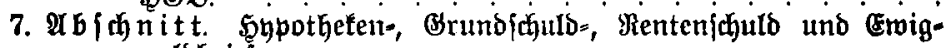
gelobriefe 


\section{Mritter đeil.}

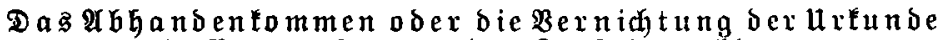
als Borauşe

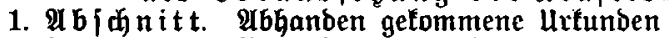

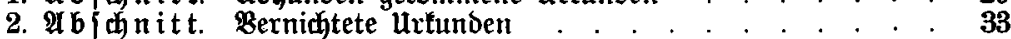

\section{Wierter aril.}

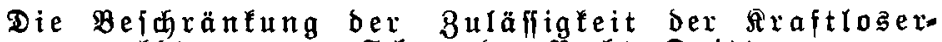

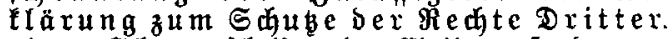

1. $\mathscr{A} b$ 出

I. Wertpapiere, für weldye Bing=, Renten= ober Betwinnan-

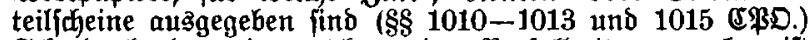

II. Equldurfunden, in reldhen eine Berfallzeit angegeben ijt (\$ 1014 (EßD.)

2. श口

\section{Jiufter aril.}

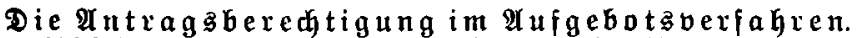

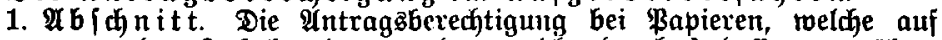

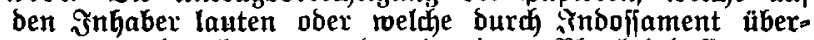
tragen werben fönnen und mit einem Blantoindoffamente verjeben find

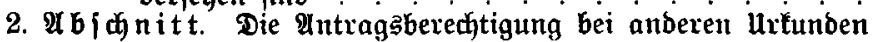

\section{Iefmater aril.}

Das $\mathfrak{A} \mathfrak{u}$ gebot $\mathfrak{g}$ exfahien.

1. $\mathfrak{A} \mathfrak{b}$ 出nitt. Borbemerkung . . . . . . . . . . . . . . 51

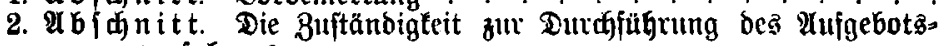
verfahrens . . . . . . . . . . . . . . . 52

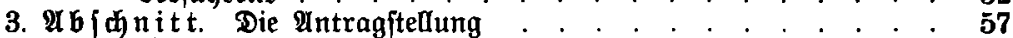

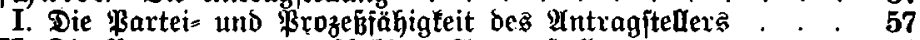

II. Die Bertretung prozeß̧fähiger : antragfteller . . . . . . . 58

III. Die Form bes $\mathscr{A}$ ntrag $\mathfrak{g}$. . . . . . . . . . . . . . . . . 59

IV. Dex Jnhalt bes Antrags .

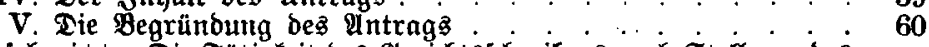

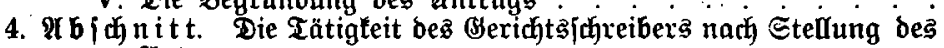
Antrags . . . . . . . . . . 63

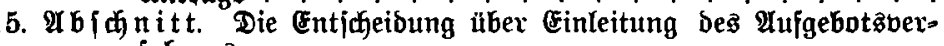
fabrens. . . . . . . . . 63

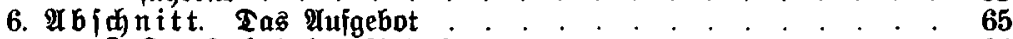

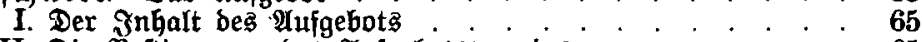

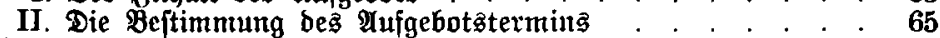

III. Die Berbffentlichung bes Ulufgebot3 . . . . . . . . . . 66

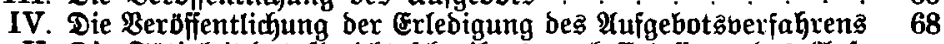

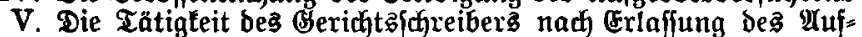
gebotz

7. $\mathfrak{A} b$ 他 gebotaberfahren . . . . . . . . . . . . . . . 69

I. Die form ber Armeldung . . . . . . . . . . . . . . 69

II. Der Beitpuntt Der $\mathscr{A n m e l}$ bung . . . . . . . . . . . . . . . . $\quad .69$

III. Dex Broed ber Âmelbung

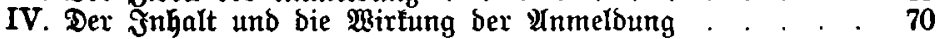


8. $\mathscr{A} \mathfrak{b} \mathfrak{d} \mathfrak{n}$ itt. Der $\mathfrak{A}$ fugebotatermin . . . . . . . . . . . 71

I. Der Bortermiu zur urtunbenuorlage . . . . . . . . . 71

II. Dex $\mathscr{A}$ ufgebotatermin . . . . . . . . . . . . . . 71

III. Das Ruhen Deă Berfahrens . . . . . . . . . . . . . . 72

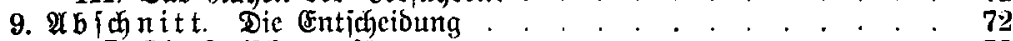

I. Die Broifchenverfügungent . . . . . . . . . . . . . . 72

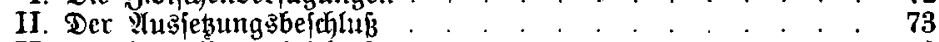

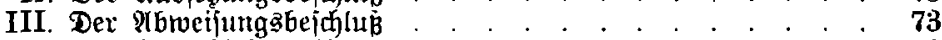

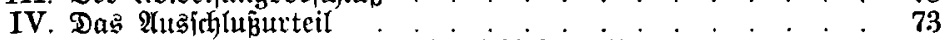

1. Die \&orausjebutg des Ausjuluburtcila . . . . . . . 73

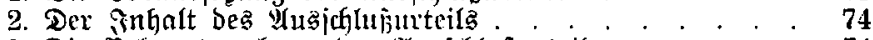

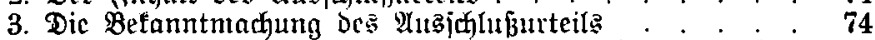

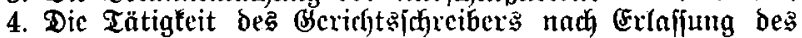

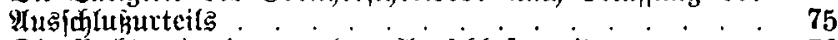

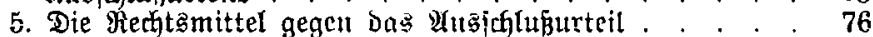

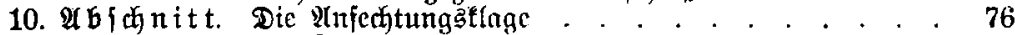

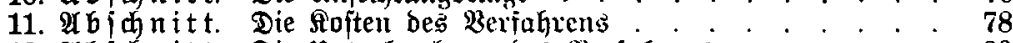

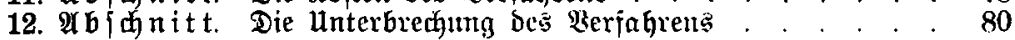

\section{Sithenter Jeil.}

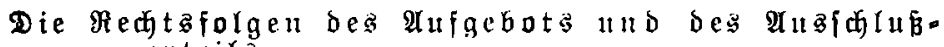
urteils.

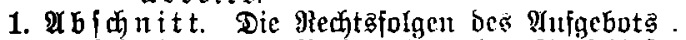

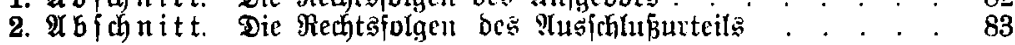

\section{Adter âtil.}

Die Bahlungajpexze.

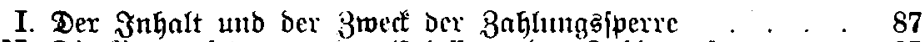

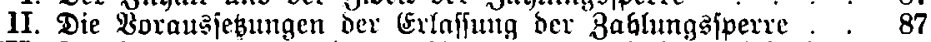

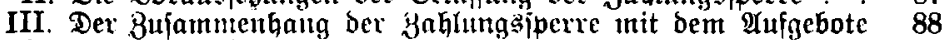

IV. Die Enticheidung über ben Rntrag auf Bahlungsiperre. 88

V. Die Bef́antmafhung Der Znhlumgäperr. . . . . . . . 88

VJ. Die Ütfebung ber 3nflungsjperre . . . . . . . . $\quad 89$

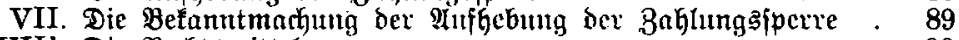

VIII. Die Rechtänittel. . . . . . . . . . . . . 90

IX. Die wirtungen ber Bahlungš̃perce . . . . . . . . . . . 90

\section{Meunter aril.}

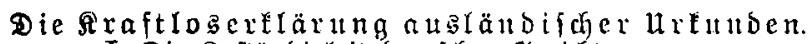

I. Die Buftäntbigfeit Deutjher Gerichte.

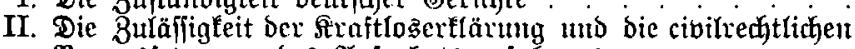

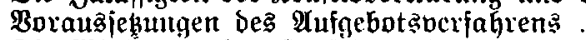

III. Die Wirtungen Der frraftloserflärung

\section{Bebuter âtil.}

Das Berbalten bei Berluft ciuer urfunde, bie im atu= gebotsoerfacen fü fraftlos extärt wexden íann.

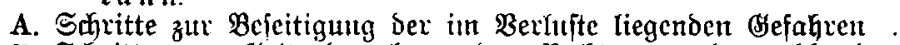

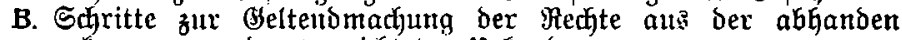
getommerten doer bernichtetcu urkube 


\section{A $\mathfrak{n} \mathfrak{a} \mathfrak{u} \mathfrak{g}$.}

I. Beifpiele für $\mathfrak{A} \mathfrak{n} \mathrm{träge}$.

Seite

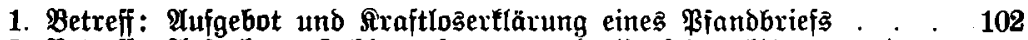

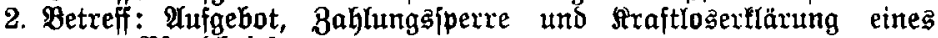
Bfanbbriefes

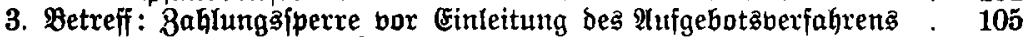

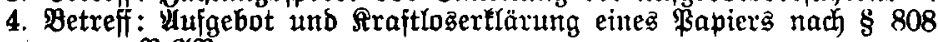
B.SBP.

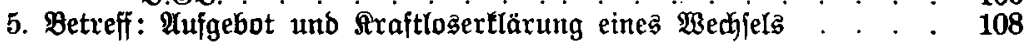

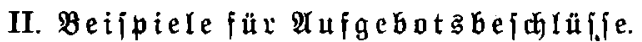

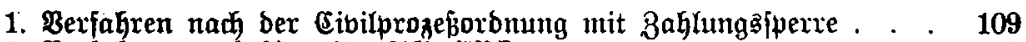

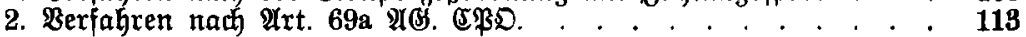

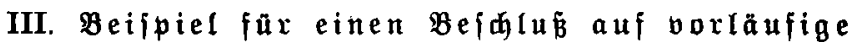

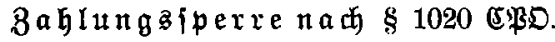

IV. Beifpiele für $\mathfrak{A} u \mathfrak{s}\{\boldsymbol{q} \mathfrak{l} \mathfrak{u} \tilde{B} \mathfrak{u}$ teile.

1. Berfałren nach ber Civilprozę̧oronung . . . . . . . . . . . 116

2. Berfahren nah $\mathfrak{A}$ rt. 69a $\mathfrak{A}$ (S). $\mathbb{P Q}$. . . . . . . . . . . . . 119

V. Beifpiele für $B$ eugniffe ber $\mathfrak{A}$ üteller.

1. Beugnia vor Einteitung bes Arfgebotsberfahrens ober vor Extaffung Der $8 \mathfrak{a}$ fungäperre .

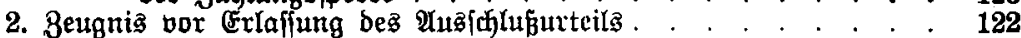

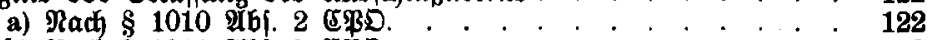

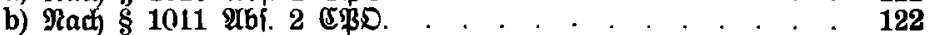

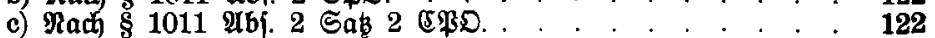

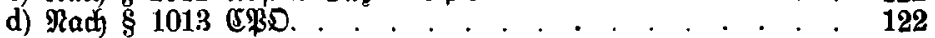

VI. (Sebú

1. Geriđtşgebühren . . . . . . . . . . . . . . . . . 124

2. Intoaltägeb̆̉̆ren . . . . . . . . . . . . . . . . . . . . 125

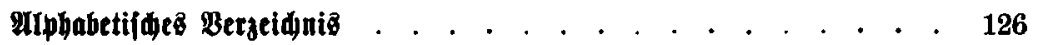

Tabellen.

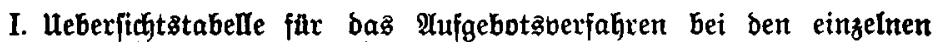
Qurten won $\mathfrak{u}$ rtunben.

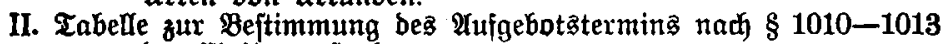
Der Cibilprozézoronung. 


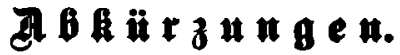

\section{Atbtiirzungen in ber Riteraturbezeiffnuag.}

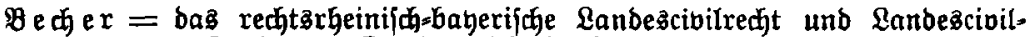

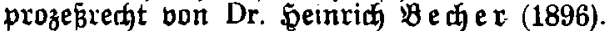

〔 oja

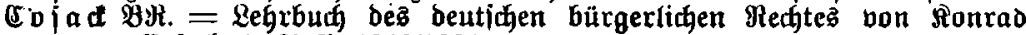
Co[act, 3. 2Aufl. 1900/1901.

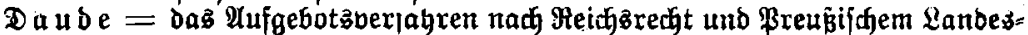
recht von Dr. B. Daude, 3. Uufl. 1900.

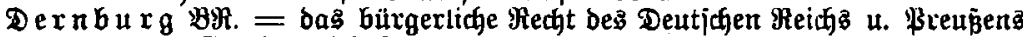
von Dr. Seinrif Dernburg, 1902.

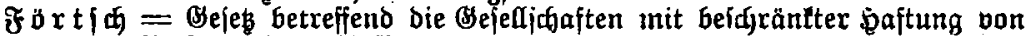

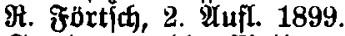

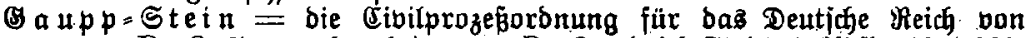
Dr. \&. Baupp, bearbeitet von Dr. Friebric Stein, 4. SÄfl. 1901/1902.

Fif

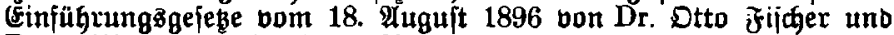

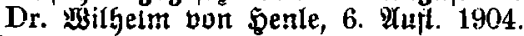

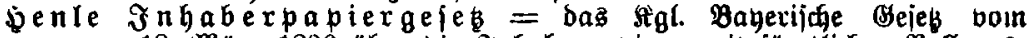

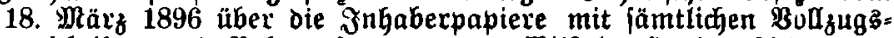

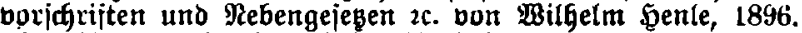

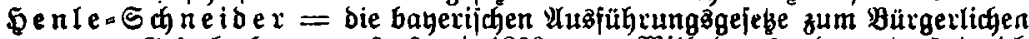

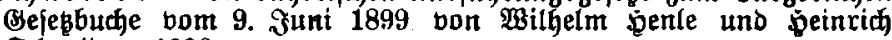
Schneiber, 1900.

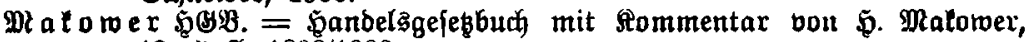
12. Uufl. $1898 / 1902$.

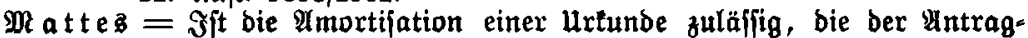
ftellex jelbft, abjichtlich ober unabjichtliç, vernichtet hat? Cin Bei=

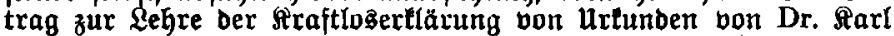
Diattez, 1899.

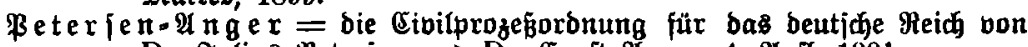

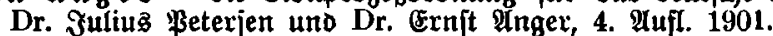

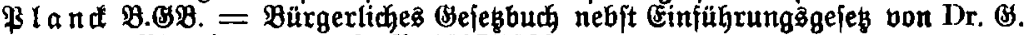
ßland, 1. u. 2. ULfl. 1897/1902.

円 е

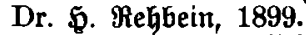

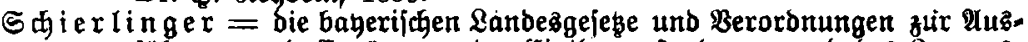
fübrung und Ergänzung ber (Eibilproze

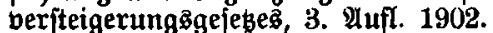

Se uffert = Jommentar zur Civilprozep̃oronung von Dr. Lothar Seuffert, 8. $\mathfrak{A}$ นโI. 1903.

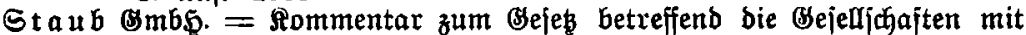

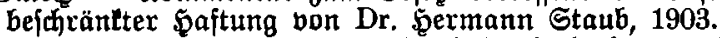

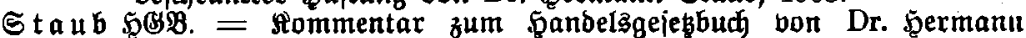
Staub, 6. น. 7. 2uful. 1900.

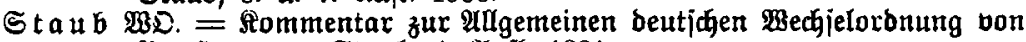
Dr. Sermarn Staub, 4. Hufl. 1901.

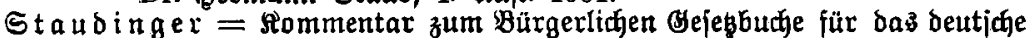

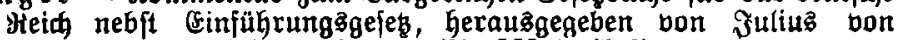
Staubinger, 1. Uufl. 1898/1903, BD. III 2. Yufl. 1903. 


\section{Spnjtige Ablïrzungent.}

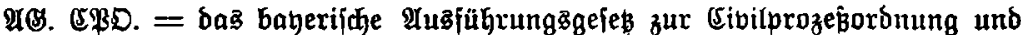
Pontursorbnung vom 23. Februar 1879 in ber Ffafjuttg bes 9 irt. 166

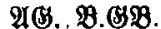

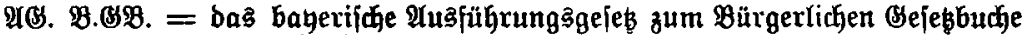
bom 9. Juni 1899.

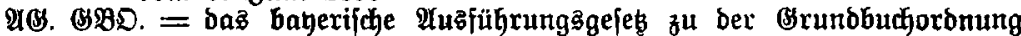

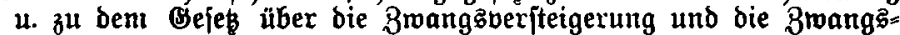
berwaltung vom 9 . Juni 1899.

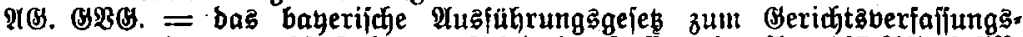

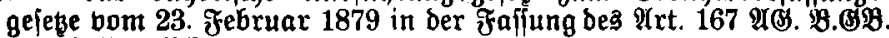

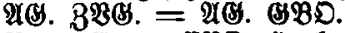

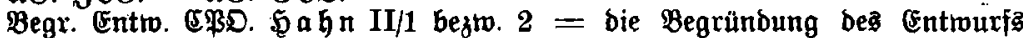
ber Cibilprozesorbnung, abgebrudt in ben gejamten Materialien

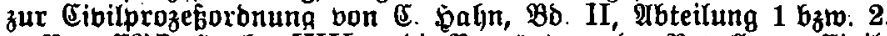

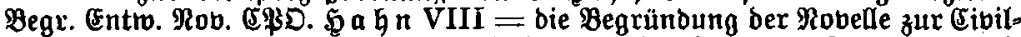
prozéporonung bom 17. Maai 1898 , abgebrudt in ben gejamten Materi=

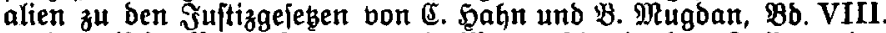

B\&. = bas bayerijue Berggeję vom 20 . März 1869 in ber Fajfung bes $\mathfrak{A}$ rt. $157 \mathfrak{A}(\mathbb{B} . \mathfrak{B}$.ङB:

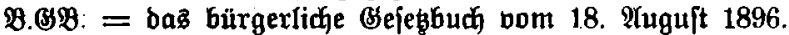

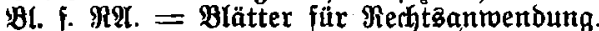

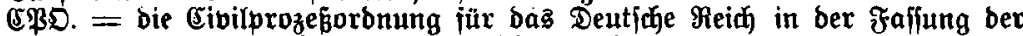
Betanntmadjung vom 20. Mai 1898 .

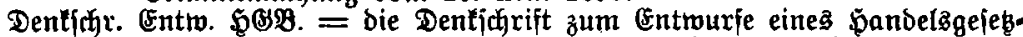

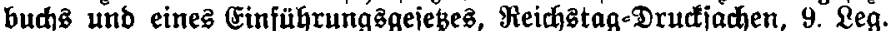
Beriobe, IV. Seifion 1895/1897 $\mathfrak{R r}$. 632 .

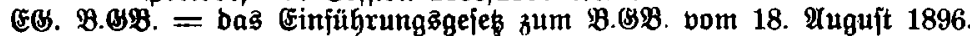

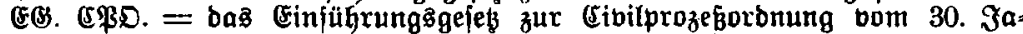
nuax 1877 in ber Fraffung ber Betanntma(f)ung bom 20. Mlai 1898.

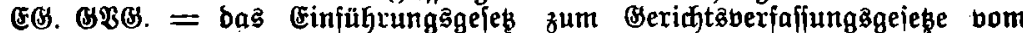
27. San. 1877 in ber Faffung ber Betanntmadhung bom 20. Mlai 1898.

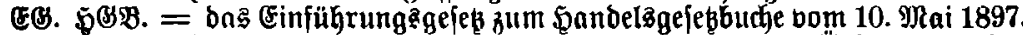

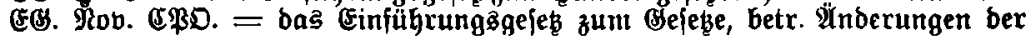
(Sibilprozeporonung bom 17. Mai 1898.

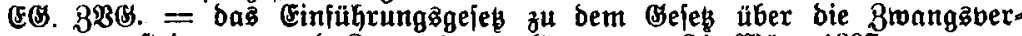
iteigerung und 3wangsverwaltung vom 24 . Mtärz 1897 .

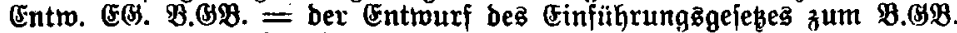

BSBD. = bie (Srunbbuthoronung bom 24. März 1897.

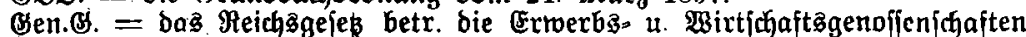
vom 1. Mtai 1889 in ber Faffung ber Betanntmachung vom 20. Mai 1898.

Beff. Anw. = bie Befanntmachung bes t. bayr. Staatzminifteriums ber

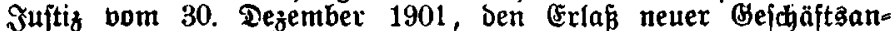

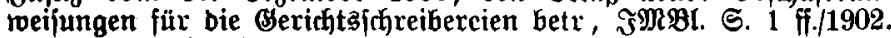

Bei.gI. = bas bayerijue Beiebesblatt.

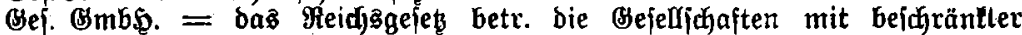
Şaftung bom 20. $\mathfrak{A}$ pril 1892 in ber Faffung ber Betantmatjung bom 20. Mai 1898.

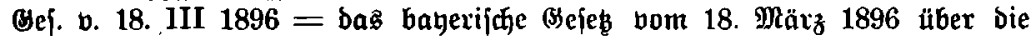
Inthaberpapiere.

(5) bom 20. Mai 1898.

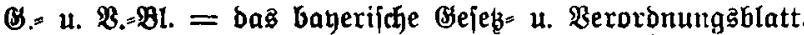

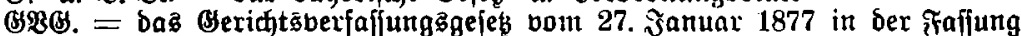
ber Betanntmachung vom 20. Mai 1898.

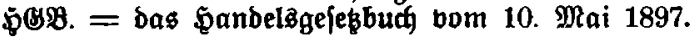




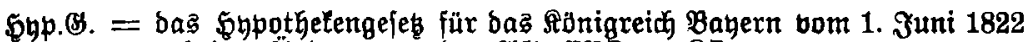

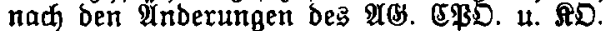

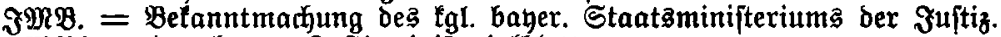

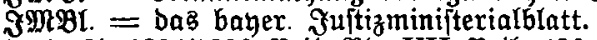

$\mathfrak{F}$. b. A. 1895/1896 Beil. Bb. VII Beil. $426=$ Berhandlungen der Rammer

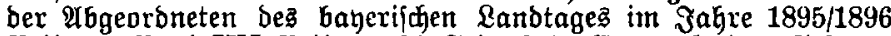
Beilagen Band VII Beilage 426 Seite 679: Entwurf eines Bejełez, einige Beftimmungen über bie Şfuldberjfhreibungen auf ben Эn= haber betr. nebit Motiven.

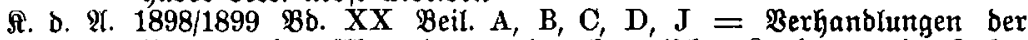
Stammex ber Mbgeoroneten dez baherifden Lanbtages in Jahre

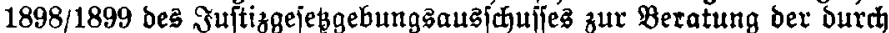

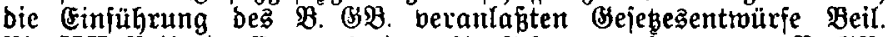

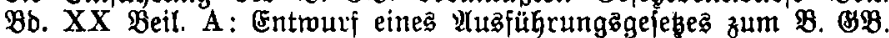
๔. 1 ff.; Beil. B: Entrurf eines BseiebeB, bie burd bie Einführung

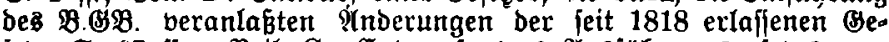
jebe $5.67 \mathrm{ff}$; Beil. C: Entruurf eines $\mathfrak{T}$ :

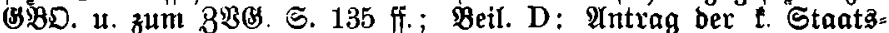
regierung zu ben Entwürfen A u. B S. 155 ff.; Beil. J : Entwurf

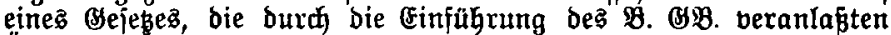
übergangsborjhriften betr. $\subseteq$. 255 fi., ie mit Begrünoung.

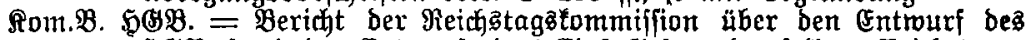

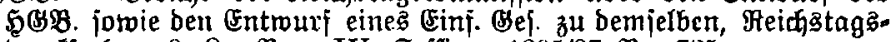
bructiađen, 9. Leg.Per., IV. Seffion, 1895/97 $\mathfrak{R x}$. 735.

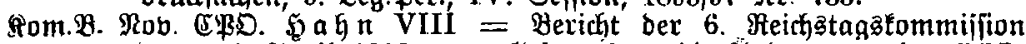
bom 26. April 1898 zum Bejeke betr bie Ânberungen Der (E\$D. bom 17. Mlai 1898 , abgebruft in ben Materialien zu ben Reids?

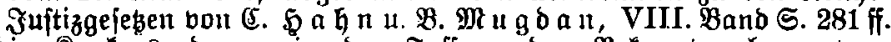

AD. = bie Ronfursoronung in ber foffung ber Betanntmachung bom 20. Mai 1898.

MPS. = Mlinijterialbefanntmanung.

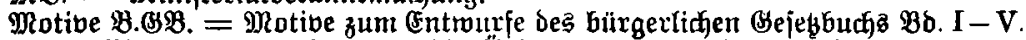

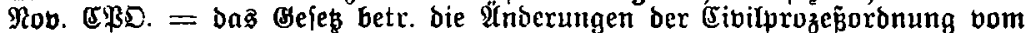
17. $\mathfrak{M} \mathfrak{a i} 1898$.

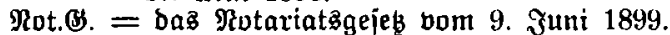

Brot Entw. B.GP. = Brotololle ber Sommijfion für die 2. Lejung beş

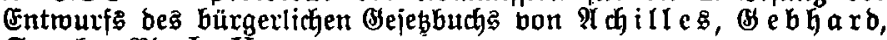
ऽ

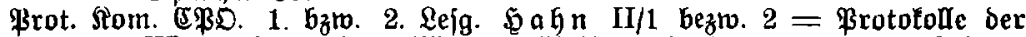

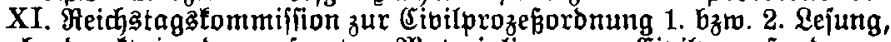
abgebrudt in ben gefamten Materialien zut Eibilprozeṕoronung von $\mathfrak{C} . \mathfrak{S} \mathfrak{a} \mathfrak{h} \mathfrak{n}, \mathfrak{B b}$. II $\mathfrak{A}$ bteilung $1 \mathfrak{b} \mathfrak{z} \mathfrak{w} .2$.

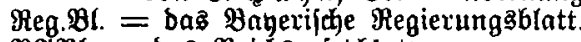

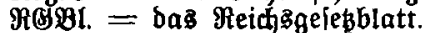

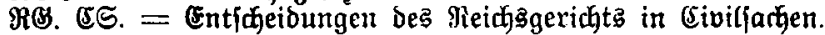

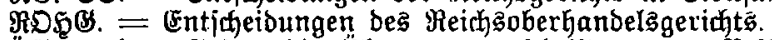

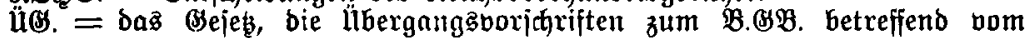
9. Эuni 1899 .

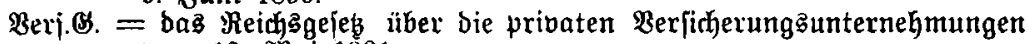
bom 12. Mai 1901.

WD. = bie 2 mgemeine beutjche Wechfeloronung

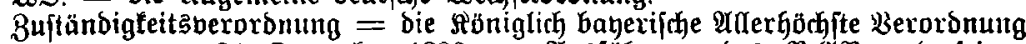

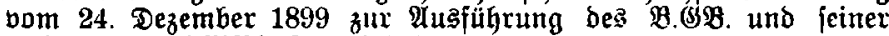

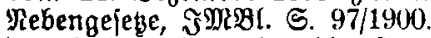

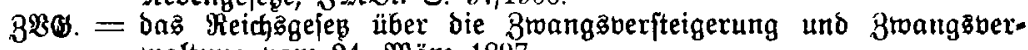
maltung bom 24. Märź 1897. 
